\title{
A single-centre experience of febuxostat as a second-line urate-lowering therapy
}

\section{Swee Gaik Ong, Hui Jen Ding}

Ong SG, Ding HJ. A single-centre experience of febuxostat as a second-line urate-lowering therapy. Malays Fam Physician. 2021;16(1);50-55. https://doi.org/10.51866/oa0892

\section{Keywords:}

febuxostat, second-line,

urate-lowering therapy, gout

\section{Authors:}

Ong Swee Gaik

(Corresponding author)

$M D, M R C P$

Rheumatology Unit, Department of

Medicine, Kuala Lumpur Hospital

Kuala Lumpur, Malaysia

Email: ongsweeg@gmail.com

\section{Ding Hui Jen}

MBChB, MRCP

Rheumatology Unit, Department of Medicine, Kuala Lumpur Hospital Jalan Pahang, Kuala Lumpur Malaysia

\section{Abstract}

Introduction: The purpose of this study was to describe the local experience in terms of drug efficacy and safety using a new xanthine oxidase inhibitor, febuxostat, as a second-line urate-lowering therapy (ULT) in gout patients with normal renal function and chronic kidney disease.

Methods: This cross-sectional study included all gout patients who attended the rheumatology clinic from January 2013 to June 2018 and had received febuxostat as a second-line ULT. Analysis focused on the proportion of gout patients who achieved target serum urate $(\mathrm{sUA})$ of $<360 \mu \mathrm{mol} / \mathrm{L}$, duration taken to achieve target sUA, and febuxostat dosage at achievement of target sUA. Safety assessments included comparison of serum creatinine, estimated glomerular filtration rate (eGFR), and serum alanine aminotransferase (ALT) at baseline, at achievement of target sUA, and at 12-monthly intervals. Results: Majority (90.9\%) of patients achieved target sUA. Median duration required to achieve target sUA was 5.5 months with IQR (interquartile range) of 8.5. Five (22.7\%) patients achieved target sUA within one month of therapy with febuxostat $40 \mathrm{mg}$ per day. Eleven $(55 \%)$ patients achieved target sUA within six months and $16(80 \%)$ by 12 months. Equal proportion of patients achieved target sUA with febuxostat $40 \mathrm{mg}$ per day and $80 \mathrm{mg}$ per day, respectively. There was no significant difference in the changes in serum creatinine level, eGFR and ALT from baseline and at achievement of target sUA, nor at 12-monthly intervals throughout the duration of febuxostat therapy. Apart from three patients who developed hypersensitivity reactions to febuxostat, no other adverse events were reported.

Conclusion: A significant proportion of gout patients with $\mathrm{CKD}$ managed to achieve target sUA with a lower dose of febuxostat at $40 \mathrm{mg}$ per day and it is reasonable to maintain this dose for up to six months before considering dose escalation.

\section{Introduction}

Gout is the most common inflammatory arthritis in adults worldwide, and if left untreated can lead to joint deformities and a poor quality of life. ${ }^{1,2}$ Over the last several decades, the incidence of gout has risen substantially, mainly due to increased frequency of comorbidities that are related to hyperuricemia, including obesity, hypertension, diabetes mellitus, dyslipidemia and chronic kidney disease (CKD). ${ }^{3-}$ 6 The growing use of drugs that induce hyperuricemia, such as thiazide and loop diuretics, is further contributing to the rise in gout cases.

Gout is attributed to hyperuricemia, which is defined as serum urate (sUA) concentration exceeding $400 \mu \mathrm{mol} / \mathrm{L}$ - the limit of urate solubility. The recommendation in gout management is to lower and maintain sUA to a level below $360 \mu \mathrm{mol} / \mathrm{L}^{7}$ in order to avoid formation of monosodium urate crystals and deposition in joints and soft tissues, thus minimizing gout flares. This treat-totarget concept is key to quality management of gout. Most gout patients require uratelowering therapy (ULT), with allopurinol being the most frequently used agent. ${ }^{8}$ It is a xanthine oxidase inhibitor that reduces urate production. Allopurinol is generally safe and well tolerated. Nevertheless, it occasionally induces serious cutaneous adverse reactions which can be potentially fatal. Patients intolerant to allopurinol would then require an alternative urate-lowering agent and the currently available option is febuxostat.

Febuxostat is a novel selective xanthine oxidase inhibitor that is used in lowering serum urate levels primarily in patients who are intolerant and non-responders to allopurinol. This drug received regulatory approval by the Ministry of Health (MOH) Malaysia in 2017. Before 2017, febuxostat use in Malaysian public hospitals required prior approval from the $\mathrm{MOH}$. To date, there is limited data on febuxostat use in Malaysian gout patients. Hence, this study aims to describe our local 
experience in terms of drug efficacy and safety using a new urate-lowering therapy, febuxostat.

\section{Methods}

This is a cross-sectional study conducted at the Rheumatology Clinic of Hospital Kuala Lumpur involving patients who received treatment from January 2013 to June 2018. Gout patients who required second-line uratelowering therapy were included. Patients were not eligible if they had not previously used allopurinol as a first-line urate-lowering agent, had incomplete medical records, had coronary heart disease, or had chronic liver disease of Child-Pugh class C. Approval from the Medical Research Ethics Committee, Ministry of Health Malaysia was obtained and registration was completed in accordance with the National Medical Research Register Malaysia (NMRR-17-2273-37871).

The target sUA was $<360 \mu \mathrm{mol} / \mathrm{L}$. Management of hyperuricemia with uratelowering therapy (ULT) was according to standard of care wherein febuxostat was initiated at $40 \mathrm{mg}$ per day, and increased to $80 \mathrm{mg}$ per day when target SUA was not attained.

The study had several objectives: examination of the proportion of gout patients who received febuxostat and achieved target sUA; assessment of the time taken to achieve target sUA; analysis of febuxostat dosage at achievement of target sUA; comparison of serum creatinine, estimated glomerular filtration rate (eGFR) using the modification of diet in renal disease (MDRD) method, ${ }^{9}$ and serum alanine aminotransferase (ALT) at baseline and at achievement of target sUA after febuxostat use.

Body mass index (BMI) according to the World Health Organization ${ }^{10}$ classification was defined as the following: underweight corresponds to BMI of $<18.5 \mathrm{~kg} / \mathrm{m}^{2}$, normal BMI value is between 18.5 to $24.9 \mathrm{~kg} / \mathrm{m}^{2}$, overweight is between 25.0 to $29.9 \mathrm{~kg} / \mathrm{m}^{2}$, and obese is $\geq 30.0 \mathrm{~kg} / \mathrm{m}^{2}$.

The current classification of chronic kidney disease $(\mathrm{CKD})$ is based on the Kidney Disease: Improving Global Outcomes (KDIGO) 2012 Clinical Practice Guideline. ${ }^{11} \mathrm{CKD}$ is defined as structural or functional abnormalities of the kidney, present for $\geq 3$ months, with or without decreased GFR, that can lead to decreased GFR; or eGFR $<60 \mathrm{ml} / \mathrm{min} / 1.73 \mathrm{~m}^{2}$ that is present for $\geq 3$ months, with or without evidence of kidney damage.

Categorical variables were reported as frequency and percentage, while continuous data were expressed as the mean (standard deviation, $\mathrm{SD}$ ) or median (interquartile range, IQR). Levels of serum creatinine, eGFR and ALT between baseline and when target SUA was achieved were analyzed using independent $\mathrm{t}$-test. Values of $\mathrm{p}<0.05$ were considered to be statistically significant. Analyses were performed using SPSS version 20.0 (IBM SPSS Inc., Chicago, IL, USA).

\section{Results}

All gout patients who received febuxostat as a second-line urate-lowering agent were included in this study. Altogether there were 24 patients. Twenty-three patients had a history of hypersensitivity reaction to allopurinol, while one had inadequate response to allopurinol. Of the 24 patients who received febuxostat, three developed hypersensitivity reaction to febuxostat wherein they experienced generalized pruritic maculopapular rash. One patient underwent successful desensitization to febuxostat and continued to receive the drug. Therefore, final analysis was undertaken on 22 patients.

Mean age of patients was 62.7 (SD 13.3) years, and $16(72.7 \%)$ were men. Sixteen $(72.7 \%)$ patients were of Malay descent, five were Chinese and one was Indian. Mean age at the onset of gout was 48.5 (SD 15.1) years. The earliest age at the onset of gout was 21 years in men compared to women at 48 years. Median duration of gout was 10 with interquartile range (IQR) of 12.8 years. Mean baseline sUA concentration was 599.5 (SD 90.6) $\mu \mathrm{mol} / \mathrm{L}$.

Mean body mass index (BMI) of the patients was 26.8 (SD 3.5) kg/m². Six (27.3\%) patients had normal BMI, 11 (50\%) were overweight, and five $(22.7 \%)$ were obese. Fifteen $(68.2 \%)$ patients had CKD and all of them were in CKD stage 3 (Table $\mathbf{1}$ ).

With regard to the concomitant use of drugs that exerted contributory effects to hyperuricemia, two patients received hydrochlorothiazide for management of hypertension, one received frusemide for treatment of pulmonary arterial hypertension, and one patient who had renal transplant received cyclosporine. 
Patient characteristics and comorbidities are depicted in Table $\mathbf{1 .}$

Table 1. Baseline demographics, gout history and comorbidities of patients.

\begin{tabular}{|c|c|}
\hline Characteristics & Patients $(n=22)$ \\
\hline Age, years & $62.7(13.3)^{\mathrm{a}}$ \\
\hline BMI, $\mathrm{kg} / \mathrm{m}^{2}$ & $26.8(3.5)^{\mathrm{a}}$ \\
\hline $\begin{array}{l}\text { Gender } \\
\text { Men } \\
\text { Women }\end{array}$ & $\begin{array}{l}16(72.7 \%) \\
6 \quad(27.3 \%)\end{array}$ \\
\hline $\begin{array}{l}\text { Ethnicity } \\
\text { Malay } \\
\text { Chinese } \\
\text { Indian }\end{array}$ & $\begin{array}{lc}16 & (72.7 \%) \\
5 & (22.7 \%) \\
1 & (4.6 \%)\end{array}$ \\
\hline Duration with gout, years & $10(12.8)^{\mathrm{b}}$ \\
\hline $\begin{array}{l}\text { Earliest age at onset of gout, years } \\
\text { Men } \\
\text { Women }\end{array}$ & $\begin{array}{l}21 \\
48\end{array}$ \\
\hline Baseline $s \mathrm{UA}, \mu \mathrm{mol} / \mathrm{L}$ & $599.5(90.6)^{\mathrm{a}}$ \\
\hline Duration of febuxostat use, months & $33.5(26.3)^{\mathrm{b}}$ \\
\hline $\begin{array}{l}\text { eGFR (MDRD), } \mathbf{n}(\%) \\
\geq 90 \mathrm{ml} / \mathrm{min} \\
60-89 \mathrm{ml} / \mathrm{min} \\
45-59 \mathrm{ml} / \mathrm{min} \\
30-44 \mathrm{ml} / \mathrm{min} \\
15-29 \mathrm{ml} / \mathrm{min} \\
<15 \mathrm{ml} / \mathrm{min}\end{array}$ & $\begin{array}{l}1(4.5 \%) \\
6(27.3 \%) \\
9(40.9 \%) \\
6(27.3 \%) \\
0(0 \%) \\
0(0 \%)\end{array}$ \\
\hline $\begin{array}{l}\text { Metabolic syndrome } \\
\text { Hypertension } \\
\text { Diabetes mellitus } \\
\text { Dyslipidemia }\end{array}$ & $\begin{array}{l}19(86.4 \%) \\
7(31.8 \%) \\
14(63.6 \%)\end{array}$ \\
\hline Family history of gout & $5(22.7 \%)$ \\
\hline Smoking & $5(22.7 \%)$ \\
\hline Alcohol use & $2(9.1 \%)$ \\
\hline $\begin{array}{l}\text { Clinical manifestations of gout } \\
\text { Tophi } \\
\text { Radiographic bone erosion } \\
\text { Medullary nephrocalcinosis } \\
\text { Urate nephrolithiasis or urolithiasis }\end{array}$ & $\begin{array}{c}19(86.4 \%) \\
9(40.9 \%) \\
5(22.7 \%) \\
0(0 \%)\end{array}$ \\
\hline
\end{tabular}

${ }^{\mathrm{a}}$ mean $(\mathrm{SD}) ;{ }^{\mathrm{b}}$ median $(\mathrm{IQR})$

Twenty (90.9\%) of 22 patients achieved target SUA (Table 2). The two patients who did not achieve target sUA had CKD. One had CKD stage $3 \mathrm{a}$ while the other had stage $3 \mathrm{~b}$. Thirteen of the 15 patients with CKD achieved target sUA. Median duration to achieve target sUA was 5.5 (IQR 8.5) months. Eleven (50\%) patients achieved target sUA within six months of commencing febuxostat. By 12 months, 16 $(72.7 \%)$ patients managed to achieve target sUA.
Five $(22.7 \%)$ patients achieved target sUA within one month of therapy. Eleven (50\%) patients reached target sUA at 6 months, $72.7 \%$ at 12 months and $90.9 \%$ at the end of study period.

Patients who had not achieved target sUA by six months with febuxostat $40 \mathrm{mg}$ per day had dose escalation to $80 \mathrm{mg}$ per day. One patient was an exception as she was commenced on febuxostat $80 \mathrm{mg}$. Eight patients (36.4\%) achieved target sUA with febuxostat $80 \mathrm{mg}$. 
Table 1. Gout history, renal function and febuxostat dosages of all patients who tolerated febuxostat

\begin{tabular}{|c|c|c|c|c|c|c|c|c|c|c|}
\hline $\mathbf{P t}$ & $\begin{array}{c}\text { Age } \\
\text { (years) }\end{array}$ & Gender & $\begin{array}{l}\text { Ethnic } \\
\text { group }\end{array}$ & $\begin{array}{c}\text { Duration } \\
\text { of gout } \\
\text { (years) }\end{array}$ & $\begin{array}{c}\text { Age at } \\
\text { onset } \\
\text { of gout } \\
\text { (years) }\end{array}$ & $\begin{array}{c}\text { Baseline eGFR } \\
\text { (MDRD) } \\
\left(\mathrm{ml} / \mathrm{min} / 1.73 \mathrm{~m}^{2}\right)\end{array}$ & $\begin{array}{c}\text { Baseline } \\
\text { sUA } \\
(\mu \mathrm{mol} / \mathrm{L})\end{array}$ & $\begin{array}{c}\text { sUA after } \\
\text { treatment } \\
\text { with } \\
\text { febuxostat } \\
(\mu \mathrm{mol} / \mathrm{L})\end{array}$ & $\begin{array}{c}\text { Duration } \\
\text { to achieve } \\
\mathrm{sUA}<360 \\
\mu \mathrm{mol} / \mathrm{L} \\
\text { (months) }\end{array}$ & $\begin{array}{c}\text { Febuxostat } \\
\text { dose to } \\
\text { achieve sUA } \\
<360 \mu \mathrm{mol} / \mathrm{L} \\
\quad(\mathrm{mg})\end{array}$ \\
\hline 1 & 67 & M & $\mathrm{M}$ & 29 & 38 & 47 & 519 & 325 & 1 & 40 \\
\hline 2 & 77 & $\mathrm{~F}$ & $\mathrm{M}$ & 13 & 64 & 52 & 518 & 287 & 1 & 40 \\
\hline 3 & 54 & $\mathrm{M}$ & C & 6 & 48 & 81 & 608 & 294 & 1 & 40 \\
\hline 4 & 67 & $M$ & I & 21 & 46 & 41 & 561 & 301 & 1 & 40 \\
\hline 5 & 63 & $\mathrm{M}$ & $\mathrm{M}$ & 10 & 53 & 39 & 516 & 272 & 1 & 40 \\
\hline 6 & 46 & $\mathrm{M}$ & $\bar{M}$ & 10 & 36 & 58 & 619 & 331 & 3 & 40 \\
\hline 7 & 74 & $\mathrm{~F}$ & M & 5 & 69 & 61 & 410 & 325 & 3 & 40 \\
\hline 8 & 58 & $\mathrm{~F}$ & $\mathrm{M}$ & 7 & 51 & 74 & 612 & 348 & 4 & 40 \\
\hline 9 & 50 & $\mathrm{~F}$ & $\mathrm{M}$ & 2 & 48 & 56 & 805 & 350 & 5 & 80 \\
\hline 10 & 85 & $\bar{M}$ & $M$ & 14 & 61 & 32 & 638 & 359 & 5 & 40 \\
\hline 11 & 63 & $M$ & C & 19 & 44 & 93 & 550 & 353 & 6 & 40 \\
\hline 12 & 33 & $M$ & $\mathrm{M}$ & 8 & 25 & 69 & 612 & 322 & 8 & 80 \\
\hline 13 & 69 & F & $\mathrm{M}$ & 5 & 64 & 60 & 735 & 346 & 9 & 80 \\
\hline 14 & 69 & $\mathrm{M}$ & $\mathrm{M}$ & 3 & 66 & 82 & 478 & 323 & 9 & 40 \\
\hline 15 & 57 & $M$ & $M$ & 24 & 33 & 39 & 698 & 358 & 10 & 80 \\
\hline 16 & 59 & $M$ & $\mathrm{M}$ & 18 & 41 & 46 & 599 & 294 & 10 & 80 \\
\hline 17 & 77 & $M$ & $M$ & 10 & 67 & 54 & 605 & 343 & 13 & 40 \\
\hline 18 & 83 & $\mathrm{~F}$ & $\mathrm{M}$ & 10 & 73 & 40 & 591 & 357 & 17 & 80 \\
\hline 19 & 68 & $\mathrm{M}$ & $\bar{C}$ & 32 & 36 & 54 & 725 & 357 & 21 & 80 \\
\hline 20 & 67 & $M$ & C & 9 & 58 & 55 & 672 & 265 & 34 & 80 \\
\hline 21 & 35 & $\mathrm{M}$ & $\mathrm{C}$ & 10 & 25 & 32 & 625 & 420 & $\mathrm{NA}$ & $\mathrm{NA}$ \\
\hline 22 & 59 & $\bar{M}$ & $M$ & 38 & 21 & 53 & 492 & 439 & NA & NA \\
\hline
\end{tabular}

Pt: patient; Gender - M: male, F: female; Ethnic group - M: Malay, C: Chinese, I: Indian; sUA: serum uric acid; eGFR (MDRD): estimated glomerular filtration rate (modification of diet in renal disease); NA: not applicable

Among the 15 patients who had CKD, 13 (86.7\%) achieved target sUA. Seven (53.8\%) of the 13 patients achieved target sUA within six months of febuxostat treatment. Of note, four of them achieved target sUA by one month. Seven (53.8\%) patients achieved target sUA with febuxostat $40 \mathrm{mg}$ while the remaining six $(46.2 \%)$ required febuxostat $80 \mathrm{mg}$.

In terms of drug safety, comparison of serum creatinine, eGFR (MDRD) and ALT was made at baseline and at achievement of target sUA. Analysis of this set of data did not show any significant difference $(p>0.05)$. Given the median duration of febuxostat use was 33.5 (IQR 26.3) months, further analysis was undertaken with regard to the comparison of serum creatinine, eGFR (MDRD) and ALT at baseline, and at every subsequent 12 months. Evaluation of these patients at 12, 24, 36 and 48 months also did not demonstrate statistically significant changes $(p>0.05)$ when compared to baseline values. None of the patients had deranged ALT values throughout the course of treatment with febuxostat.

Febuxostat was generally well tolerated.
Majority of patients who were switched to febuxostat did not develop hypersensitivity reaction (23/24 were allergic to allopurinol, while $3 / 24$ were allergic to febuxostat). No other adverse effects were reported. Nonetheless, two patients were advised to discontinue febuxostat at seven months and 48 months of treatment. One of them was diagnosed to have coronary heart disease from coronary angiography several months after commencement of febuxostat. Coronary angiography was carried out during her workup for pulmonary arterial hypertension. The other patient was later found to have sonographically-confirmed liver cirrhosis given the history of chronic alcohol consumption.

\section{Discussion}

Even though allopurinol is recommended as the first-line ULT for the management of gout, ${ }^{12}$ its use has occasionally been hampered by the development of adverse events, in particular, serious cutaneous adverse reactions in certain patients. Studies have shown that a significant proportion of gout patients have concomitant $\mathrm{CKD},{ }^{13}$ which impacts the management of gout. Given the general 
understanding that allopurinol requires dose adjustment according to creatinine clearance, coupled with the fact that the safest and most effective dosing of allopurinol is still debatable, it is not unexpected for patients with gout and $\mathrm{CKD}$ to be prescribed suboptimal doses of allopurinol. ${ }^{14}$ This inevitably leads to failure in achieving target sUA, culminating in greater frequency of gout flares among CKD patients. With the advent of febuxostat, an alternative ULT can be offered to patients who are intolerant to, or have failed to respond to, allopurinol.

A vast majority of the patients in our cohort achieved target sUA, a finding which concurs with previous studies confirming the effectiveness of febuxostat. ${ }^{16-18}$ The fact that five of the 22 patients in our cohort achieved target sUA within one month of febuxostat therapy and seven achieved target sUA by three months of therapy proved that febuxostat is indeed a potent ULT. In addition, 10 of the 11 patients who achieved target sUA within six months of febuxostat treatment were in fact receiving a lower dose of febuxostat at $40 \mathrm{mg}$ per day. Among the CKD patients in our cohort, there was an almost equal proportion who responded to febuxostat $40 \mathrm{mg}$ and $80 \mathrm{mg}$ per day, respectively. This observation demonstrated that a lower dose of febuxostat can be effective in patients with CKD. Hence, it would be prudent to initiate febuxostat at $40 \mathrm{mg}$ per day instead of $80 \mathrm{mg}$ per day in patients with mild to moderate renal impairment. In the event the target sUA is not achieved by six months, febuxostat dose can be escalated to $80 \mathrm{mg}$ per day. Interestingly, the authors would like to highlight that two patients (Patients 14 and 17 in Table 2) who received febuxostat $40 \mathrm{mg}$ per day eventually achieved target sUA, albeit at 9 months and 13 months, respectively. The reason why both patients remained on febuxostat $40 \mathrm{mg}$ per day without dose escalation was because they encountered logistics issues, rendering them unable to attend clinic appointments at earlier dates. Of note, the usual dosing regime for febuxostat is $40 \mathrm{mg}$ per day with dose escalation to $80 \mathrm{mg}$ after two to four weeks.

With regard to drug safety, there were no major adverse events reported in our cohort. Liver and renal functions of our cohort were not significantly affected throughout the course of febuxostat therapy. Nevertheless, we had to discontinue febuxostat in one patient when sonographically-evident liver cirrhosis was detected, given the fact that febuxostat is predominantly metabolised in the liver. We concluded that the aetiology of liver cirrhosis was most likely attributed to alcohol and not febuxostat, as that patient had a longstanding history of alcohol consumption. ${ }^{19}$ Notwithstanding the fact that he had received febuxostat for 48 months, his ALT had remained within the normal range throughout this period. Upon further consideration, the decision to discontinue febuxostat may not be valid as our patient had chronic liver disease of Child-Pugh class A. According to Khosrawan et $\mathrm{al}^{20}$ the pharmacokinetics of febuxostat were unaffected in patients with mild-to-moderate hepatic impairment i.e. Child-Pugh classes A and $B$, thus suggesting that febuxostat is only contraindicated in Child-Pugh class C.

Another patient correspondingly had febuxostat terminated at the seventh month of therapy after she was diagnosed to have coronary heart disease in the course of investigation for pulmonary arterial hypertension. This decision was made based on the findings in the CARES (cardiovascular safety of febuxostat and allopurinol in patients with gout and cardiovascular morbidities) trial that demonstrated an adverse impact of febuxostat on all-cause mortality as well as cardiovascular mortality in gout patients with coexisting cardiovascular disease, although the mechanism underlying the mortality risk remains unclear. ${ }^{21}$

Despite the encouraging findings, this study has its limitations given the small sample size. Therefore, caution should be exercised in interpreting our findings as this study may be statistically underpowered. Given the fact that current gout guidelines recommend for febuxostat to be used as a second-line uratelowering agent, it is not unexpected that this would be the limiting factor in determining the sample size. We anticipate readers might question why other trials involving febuxostat had enormous sample sizes while our sample size was substantially smaller. We would therefore like to remind readers that those trials were comparative trials using allopurinol vs febuxostat as first-line urate-lowering agents. The second limitation is that this research did not compare the urate-lowering effect, safety and tolerability of febuxostat with allopurinol.

\section{Conclusion}

Our study showed that a significant proportion of gout patients with CKD managed to achieve 
target sUA with a lower dose of febuxostat at $40 \mathrm{mg}$ per day. We suggest that this dose be maintained for up to six months in the event that target sUA has not been achieved before considering dose escalation.

\section{Funding}

This research did not receive any funding from any agency.

\section{Conflict of interest statement}

The authors declared no potential conflicts of interest with respect to the research, authorship, and/or publication of this article.

\section{Acknowledgment:}

The authors would like to thank the DirectorGeneral of Health Malaysia for the permission to publish this paper.

\section{References}

1. Roddy E, Zhang W, Doherty M. Is gout associated with reduced quality of life? A casecontrol study. Rheumatology 2007;46(9):1441-4.

2. Singh JA, Strand V. Gout is associated with more comorbidities, poorer healthrelated quality of life and higher healthcare utilisation in US veterans. Ann Rheum Dis 2008;67(9):1310-6

3. Wallace KL, Riedel AA, Joseph-Ridge N, et al. Increasing prevalence of gout and hyperuricemia over 10 years among older adults in a managed care population. J Rheumatol 2004;31(8):1582-7.

4. Klemp P, Stansfield SA, Castle B, et al. Gout is on the increase in New Zealand. Ann Rheum Dis 1997;56(1):22-6.

5. Nan H, Qiao Q, Dong Y, et al. The prevalence of hyperuricemia in a population of the coastal city of Qingdao, China. J Rheumatol 2006;33(7):1346-50.

6. Miao Z, Li C, Chen Y, et al. Dietary and lifestyle changes associated with high prevalence of hyperuricaemia and gout in the Shandong coastal cities of Eastern China. J Rheumatol 2008;35(9):1859-64.

7. Bardin T. Hyperuricemia starts at 360 micromoles (6 mg/dL). Joint Bone Spine 2015; 82(3):141-3.

8. Khanna D, Fitzgerald JD, Khanna PP, et al. 2012 American College of Rheumatology guidelines for management of gout. Part 1: Systematic nonpharmacologic and pharmacologic therapeutic approaches to hyperuricemia. Arthritis Care Res (Hoboken) 2012;64(10):1431-46.
9. Stevens LA, Coresh J, Feldman HI, et al. Evaluation of the modification of diet in renal disease study equation in a large diverse population. J Am Soc Nephrol. 2007;18(10):2749-57.

10. International Obesity Task Force. Managing the Global Epidemic of Obesity. Report of the World Health Organization (WHO) Consultation on Obesity; June 5-7, 1997; Geneva, Switzerland.

11. Kidney disease: Improving global outcomes (KDIGO) CKD work group. KDIGO 2012 Clinical practice guideline for the evaluation and management of chronic kidney disease. Kidney Int Suppl. 2013(1):1-150.

12. Richette P, Doherty M, Pascual E, et al. 2016 updated EULAR evidence-based recommendations for the management of gout. Ann Rheum Dis 2017;76:29-42.

13. Fuldeore MJ, Riedel AA, Zarotsky V, et al. Chronic kidney disease in gout in a managed care setting. BMC Nephrol 2011;12:36.

14. Dalbeth N, Kumar S, Stamp L, et al. Dose adjustment of allopurinol according to creatinine clearance does not provide adequate control of hyperuricemia in patients with gout. J Rheumatol 2006;33(8):1646-50.

15. Becker MA, Schumacher HR, Wortmann RL, et al. Febuxostat compared with allopurinol in patients with hyperuricemia and gout. NEJM 2005;353(23):2450-61

16. Schumacher HR, Becker MA, Lloyd E, et al. Febuxostat in the treatment of gout: 5-yr findings of the FOCUS efficacy and safety study. Rheum 2009;48(2):188-94.
17. Becker MA, Schumacher HR, Espinoza LR, et al. The urate-lowering efficacy and safety of febuxostat in the treatment of the hyperuricemia of gout: the CONFIRMS trial. Arthritis Res Ther 2010;12(2):R63.

18. Saag KG, Whelton A, Becker MA, et al. Impact of febuxostat on renal function in gout patients with moderate-to-severe renal impairment. Arthritis Rheum 2016;68(8):2035-43.

19. Sorensen TI, Orholm M, Bentsen KD, et al Prospective evaluation of alcohol abuse and alcoholic liver injury in men as predictors of development of cirrhosis. Lancet. 1984:2:241-244.

20. Khosravan R, Grabowski BA, Mayer $\mathrm{MD}$, et al. The effect of mild and moderate hepatic impairment on pharmacokinetics, pharmacodynamics, and safety of febuxostat, a novel nonpurine selective inhibitor of xanthine oxidase, J Clin Pharmacol 2006;46(1):88-102.

21. White WB, Saag KG, Becker MA, Borer JS, et al. Cardiovascular safety of febuxostat or allopurinol in patients with gout. NEJM 2018; 378(13):1200-10. 